\title{
Dense Glass Packing Can Slow Reactions with an Atmospheric Gas
}

\author{
Yue Qiu, ${ }^{*(1)}$ Michael E. Bieser, and M. D. Ediger ${ }^{\circledR}$ \\ Department of Chemistry, University of Wisconsin-Madison, Madison, Wisconsin 53706, United States
}

\section{Supporting Information}

\begin{abstract}
Previous work utilizing crystal polymorphs has established the importance of the molecular packing environment for modulating solid-gas reactivity. Here, we show, for the first time, that the chemical stability of an amorphous material in contact with a reactive gas can be significantly improved by controlling glass packing. We utilize the reaction of indomethacin with ammonia as this system has been well-characterized for crystalline polymorphs. For these experiments, physical vapor deposition (PVD) is used to prepare glasses of indomethacin with a range of densities and thermal stabilities. The indomethacin-ammonia reactivity is assessed through the increase in mass of glassy thin films exposed to ammonia gas, as characterized by a quartz crystal microbalance. Indomethacin glasses vapor-deposited at substrate temperatures below the glass transition temperature $\left(T_{\mathrm{g}}\right)$ show unprecedented decrease in reaction rates relative to the liquid-cooled glass, by as much as 1 order of magnitude, with the densest glasses having the slowest reactions. The diminished solubility of ammonia in dense PVD glasses is found to be a major factor in their remarkable chemical stability. As chemically stable amorphous solids are in demand for applications including pharmaceuticals and organic electronics, this work provides a strategy to improve performance of these materials.
\end{abstract}

\section{INTRODUCTION}

Organic glasses are important materials that are widely used in modern technologies, including pharmaceuticals ${ }^{1,2}$ and organic electronics. ${ }^{3-5}$ In comparison to their crystalline counterparts, organic glasses are frequently preferred in many applications. For example, some drugs are formulated as glasses because of higher solubility and bioavailability. ${ }^{6}$ In the organic light emitting diode (OLED) industry, glassy thin films are used as light emission layers because they have smooth surfaces and readily accommodate mixtures while lacking grain boundaries. One important issue for organic materials is their reactivity with environmental gases. For example, several drugs are known to undergo hydrolysis reactions with water vapor, leading to shortened shelf life. ${ }^{7,8}$ For OLEDs, it has been reported that ambient gases, such as water vapor and oxygen, have a significant negative impact on the device lifetime. ${ }^{9-11}$

Work dating back more than 50 years has established that local packing arrangements in crystals can modulate chemical reactivity with gases. ${ }^{12,13}$ It has been found that gases typically react with crystals through a growth front mechanism, with reactions starting at the crystal surface. As tight molecular packing does not readily allow diffusion of gases into the crystal, gases react with crystals at a facet with exposed functional groups or at a defect site. Once reactions are initiated from the surface, the crystal lattice is disrupted, allowing reaction with the next layer of the crystal. This process can happen over and over again, resulting in a reaction front moving into the crystal at a constant velocity; such reactions show zero order kinetics with the reaction rate depending upon the amount of exposed surface area. The largest reaction rate will occur at the facet that provides the easiest access for gas molecules to exposed functional groups, and thus, the crystal/gas reaction can be highly anisotropic.

One of the best-studied cases of crystal/gas reactions is the reaction of ammonia with indomethacin, a drug molecule that contains a carboxylic acid group (Scheme 1). The $\alpha$ crystal

Scheme 1. Indomethacin Reaction with Ammonia<smiles>COc1ccc2c(C(O)c3ccc(OCC(C)C)cc3)n(C(=O)c3ccc(Cl)cc3)c(CC(=O)O)c2c1</smiles>

polymorph of indomethacin readily reacts with ammonia and shows a surface-initiated anisotropic reaction mechanism, consistent with the features described in the previous paragraph. ${ }^{13}$ A further study compared the reactivity of $\alpha$ indomethacin with the $\gamma$ polymorph. Remarkably, the $\gamma$ polymorph reacts at least 100 times more slowly than the $\alpha$ polymorph, ${ }^{14}$ even though the $\gamma$ polymorph is $4 \%$ less dense. ${ }^{12}$ Chen et al. explained this result by noting that the carboxylic acid groups in the $\gamma$ polymorph are shielded by a hydrophobic

Received: September 3, 2019

Revised: October 31, 2019

Published: November 6, 2019 
shell that blocks access to ammonia molecules. ${ }^{14}$ For crystals of a broad range of different carboxylic acids reacting with ammonia, reaction rates are not correlated with acid dissociation constant, crystal density, or melting point. ${ }^{15}$ Rather, it is the detailed local packing arrangements in the crystal that control reactivity.

In contrast to crystals, we know much less about how chemical reactions proceed when glasses are exposed to reactive gases. As glasses do not have faceted surfaces or long range orders, one would anticipate that different glasses of the same molecule would react very similarly with any particular gas. Indeed, we are not aware of any report of a glass-gas reaction in which different glasses of a given molecule show a significant modulation in chemical reactivity.

Recently, physical vapor deposition (PVD) has been reported to prepare "stable glasses" with exceptional properties that are not accessible by any other preparation method. ${ }^{16}$ By properly controlling the substrate temperature during deposition, vapor deposition can form glasses that have much higher thermal stability relative to that of traditional liquid-cooled (LC) glasses. ${ }^{16-20}$ In comparison with LC glasses, PVD glasses also have higher densities ${ }^{18}$ and lower enthalpies. ${ }^{17,21}$ With respect to both density and enthalpy, the most stable PVD glasses have moved about $25 \%$ of the way from the LC glass to the crystal. ${ }^{19}$ These are much larger changes than can be practically accomplished by aging a LC glass or forming the glass by more slowly cooling the liquid.

The discovery of PVD stable glasses provides a unique opportunity to address fundamental questions about chemical reactions in glasses. ${ }^{22}$ In the present work, we return to indomethacin as a model system because the reactivity of its crystalline polymorphs has been well characterized. ${ }^{14}$ Indomethacin has been found to form high-density and lowenthalpy glasses by vapor deposition, with the density increased as much as $1.4 \%$ and the enthalpy decreased by up to $4.3 \mathrm{~kJ} / \mathrm{mol}$ relative to the LC glass. ${ }^{18,19}$ Here we investigate the reaction rate when PVD and LC glasses of indomethacin are exposed to ammonia gas. In this work, indomethacin glasses with different densities and local packing arrangements were obtained by vapor deposition onto substrates held at different temperatures. A quartz crystal microbalance (QCM) was used to continuously monitor the mass gain induced by reaction with ammonia. The influences of ammonia concentration, film thickness, and reaction temperature on reactivity were investigated.

We find that PVD indomethacin glasses can be significantly more stable against reaction with ammonia gas than the LC glass. For reaction at $295 \mathrm{~K}$, the glass vapor-deposited at $260 \mathrm{~K}$ $\left(0.84 T_{\mathrm{g}}\right)$ reacts at one-tenth the rate of the LC glass. This is the first demonstration that glass packing can significantly modulate the rate of a solid-gas reaction. We observe that chemical stability is strongly correlated with the glass density. In contrast to crystalline indomethacin, the reaction of ammonia with glassy indomethacin does not proceed from the free surface and is not limited by gas diffusion. We conclude that decreased solubility of ammonia in dense glasses is likely a major factor in explaining the slower reaction rate.

\section{EXPERIMENTAL METHODS}

2.1. Preparation of PVD Indomethacin Glasses. Indomethacin (99\%) was purchased from Sigma-Aldrich and used as received. Glassy thin films of indomethacin were prepared by PVD, which took place in a vacuum chamber
( $10^{-7}$ Torr). Indomethacin was placed in a crucible that was resistively heated to sublime the material into the vapor phase. The deposition rate was controlled by tuning the heating power and monitored by a QCM. For all the samples prepared for this paper, the deposition rate was kept at a constant value of $0.2 \mathrm{~nm} / \mathrm{s}$. Indomethacin was directly deposited onto the substrate of a gold-coated quartz crystal resonator, which is suitable for use in the QCM measurement that will be introduced below. During vapor deposition, the quartz crystal resonator was in good thermal contact with a copper finger, whose temperature was precisely controlled. For these experiments, most samples were about $100 \mathrm{~nm}$ in thickness, while $300 \mathrm{~nm}$ samples were used in a few experiments. LC glasses were prepared by vapor deposition at a substrate temperature of $320 \mathrm{~K}$, which is $10 \mathrm{~K}$ above the $T_{\mathrm{g}}\left(T_{\mathrm{g}}=310\right.$ $\mathrm{K})$, followed by cooling to room temperature at $1 \mathrm{~K} / \mathrm{min}^{18}$

2.2. Characterization of Reaction Kinetics. The indomethacin-ammonia reaction was performed in a custom-built reaction chamber. Vapor-deposited indomethacin on the quartz crystal resonator was placed in a QCM device, located within the reaction chamber. Dilute ammonia gas was prepared by bubbling nitrogen through ammonium hydroxide solutions at a flow rate of $10 \mathrm{~mL} / \mathrm{min}$. The gas flowed through a desiccating tube of potassium hydroxide pellets to remove water vapor before entering the reaction chamber. In this paper, ammonium hydroxide solutions of two concentrations were used to generate ammonia gas, that is, $0.49 \mathrm{M}$ (1 wt \%) and $1.5 \mathrm{M}$ (3 wt \%). For the flow conditions utilized, the ammonia gas in the ammonia-nitrogen mixture had a partial pressure of $0.0076 \pm 0.0007$ and $0.025 \pm 0.004$ bar for these two solutions, respectively.

Prior to each reaction, the sample in the reaction chamber was exposed to a stream of pure nitrogen gas. To initiate the reaction, the pure nitrogen was turned off and the ammonianitrogen mixture was directed into the reaction chamber. For most experiments, the reaction was allowed to proceed for 15 min before turning off the ammonia gas. In some experiments, after the initial exposure of ammonia gas, the stream of pure nitrogen gas was turned on again at a flow rate of $2000 \mathrm{~mL} /$ min to purge the sample (typically for $15 \mathrm{~min}$ ), followed by a second exposure to ammonia gas. For some indomethacin samples, this procedure was utilized several times to acquire reaction data for multiple cycles of exposure. Most experiments were performed at room temperature $(295 \mathrm{~K})$. For lower temperature experiments, the reaction chamber was cooled to $275 \pm 1 \mathrm{~K}$.

The mass uptake during the addition reaction of ammonia was monitored by a QCM. As described above, indomethacin glasses were directly deposited onto a $5 \mathrm{MHz}$ AT-cut quartz plate (Inficon Inc.) with a polished gold electrode that was utilized as the QCM resonator. QCM is widely used as a sensitive mass detector in gas uptake measurements. ${ }^{23,24}$ For films in the thickness range employed here, the frequency shift of the resonator can be related to the mass change of the sample by the Sauerbrey equation ${ }^{25}$

$$
\Delta f=-\frac{2 f_{0}^{2}}{A \sqrt{\rho_{\mathrm{q}} \mu_{\mathrm{q}}}} \Delta m
$$

where $f_{0}$ is the resonance frequency $(\mathrm{Hz}), \Delta f$ is the frequency change, $\Delta m$ is the mass change, $A$ is the piezoelectrically active crystal area, $\rho_{\mathrm{q}}$ is the density of quartz $\left(2.648 \mathrm{~g} / \mathrm{cm}^{3}\right)$, and $\mu_{\mathrm{q}}$ is the shear modulus of quartz for the AT-cut crystal $(2.947 \times$ 
$\left.10^{11} \mathrm{~g} \cdot \mathrm{cm}^{-1} \cdot \mathrm{s}^{-2}\right)$. Because $f_{0}, A, \rho_{\mathrm{q}}$, and $\mu_{\mathrm{q}}$ are all known, $\Delta m$ can be calculated from $\Delta f$. In this way, we can monitor the indomethacin-ammonia reaction of the glassy thin films in real time.

\section{RESULTS AND DISCUSSION}

3.1. Influence of the Glass Structure on Reactivity. Figure 1 shows the comparison of the mass increase during the

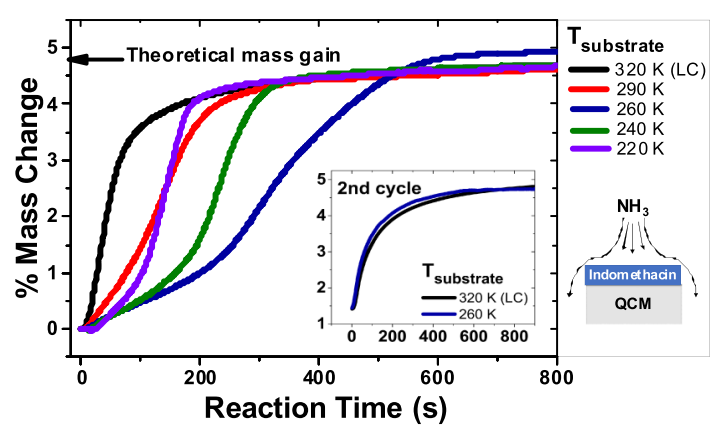

Figure 1. Mass increase for $100 \mathrm{~nm}$ thick indomethacin glasses reacting with 0.0076 bar ammonia (in nitrogen) as a function of time at $295 \mathrm{~K}$. The colored curves represent glasses vapor-deposited at the indicated substrate temperatures. The black curve represents the LC glass (deposited above $T_{\mathrm{g}}$ at $320 \mathrm{~K}$ and then cooled at $1 \mathrm{~K} / \mathrm{min}$ ). The inset shows the 2nd cycle of the reaction after glasses have reacted with ammonia and have been purged with $\mathrm{N}_{2}$ gas. Schematic in the lower right shows the device geometry used to measure the kinetics of the indomethacin-ammonia reaction.

reaction with ammonia gas for PVD and LC indomethacin glasses, and reveals that the PVD glasses display a slower reaction, that is, enhanced chemical stability. These tests were performed on $100 \mathrm{~nm}$ films reacting with 0.0076 bar ammonia at $295 \mathrm{~K}$. Immediately after ammonia gas is introduced, the mass starts to increase for all glasses. After $600 \mathrm{~s}$, the mass of all the glasses approximately reaches a steady-state, and the overall mass increase at steady-state is in the range of $4.6-4.9 \%$. The observed mass gain is reasonably consistent with the theoretical value of $4.8 \%$ calculated from the reaction stoichiometry, indicating that nearly all indomethacin molecules have reacted. This provides confidence that QCM can precisely characterize the chemical reaction occurring in glassy indomethacin. We performed IR measurements to confirm that the mass increase shown in Figure 1 is correlated with the disappearance of absorption peaks associated with the carboxylic acid group of indomethacin (Figure S1).

We found that the distinctive packing of glasses vapordeposited at different substrate temperatures was destroyed as a result of reaction with ammonia gas. After reaching steadystate in the initial exposure to ammonia, we purged the system with pure nitrogen gas (shifting the equilibrium and removing about $3 / 4$ of the reacted ammonia) and performed a second cycle of the ammonia reaction. As illustrated in the inset of Figure 1, for the second cycles of the reaction, indomethacin glasses vapor-deposited at 260 and $320 \mathrm{~K}$ show very similar mass increases. Thus, the initial glass packing has been completely disrupted by the first round of reaction with ammonia. We confirmed this with wide-angle X-ray scattering measurements on PVD glasses before and after the first exposure to ammonia. Glasses deposited at $T_{\text {sub }}=260 \mathrm{~K}$ show an anisotropic peak $\left(q=0.57 \AA^{-1}\right)$ that indicates a tendency for molecular layering, ${ }^{26}$ but this peak is destroyed by the reaction with ammonia (Figure S2), revealing that the initial structure of the as-prepared glasses is substantially perturbed.

To compare quantitatively the chemical stability of PVD and LC glasses of indomethacin, we summarize the reaction halflife $\left(t_{1 / 2}\right)$ for each glass in Figure 2a. The half-life is defined as

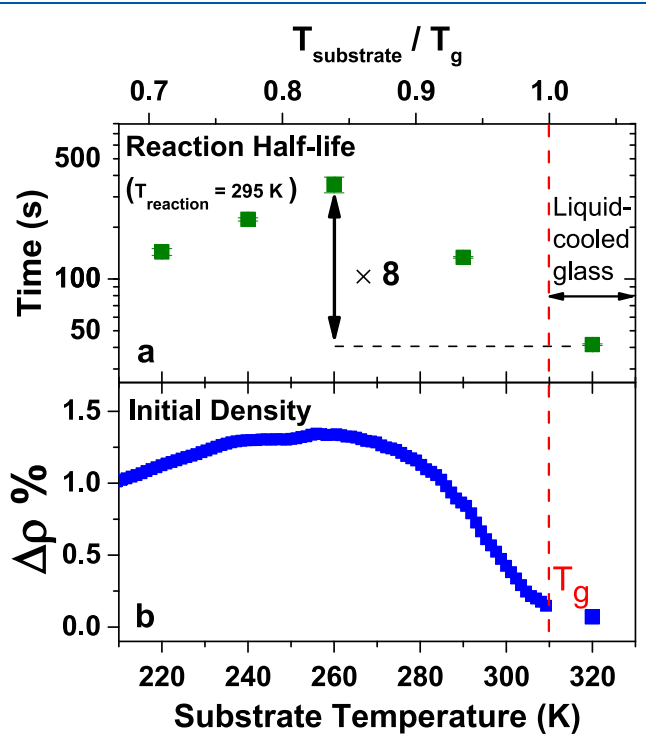

Figure 2. Reaction half-lives for the indomethacin glass-ammonia gas reaction (a) and indomethacin glass density (b), as a function of substrate temperature during vapor deposition. The density of the asdeposited glasses is presented relative to the LC glass; data are replotted from ref 18. A good correlation is observed between glass density and resistance to the reaction with ammonia. Error bars in panel a represent the standard deviation of at least two experiments.

the time required to transform half of the indomethacin in a given sample into ammonium salt. For these reaction conditions, the half-life for the LC glass is about $40 \mathrm{~s}$, while all the glasses vapor-deposited at $290 \mathrm{~K}$ and below have $t_{1 / 2}$ at least 3 times longer than this, indicating enhanced chemical stability. The most stable PVD glass employed, which was prepared at a substrate temperature of $260 \mathrm{~K}$, has a $t_{1 / 2}$ about 8 times longer than the LC glass.

The reaction half-lives of PVD and LC glasses are compared with the initial glass density in Figure $2 a, b$, and a good correlation between reaction half-life and glass density can be observed. Figure $2 \mathrm{~b}$ shows the density of indomethacin glasses vapor-deposited at different temperatures relative to the LC glass. All glasses prepared at $T_{\text {sub }}<T_{\mathrm{g}}$ investigated in this study show higher density and diminished reaction rates with ammonia.

The correlation between indomethacin glass density and reaction half-life shown in Figure 2 contrasts strongly with the reactivity of the crystal polymorphs of indomethacin. For indomethacin, the higher reaction rate occurred in the denser polymorph. Of the two polymorphs of indomethacin with millimeter-sized crystals, the $\alpha$-form finished the reaction with ammonia after $5 \mathrm{~min}$, while the $\gamma$-form barely had any change even after $24 \mathrm{~h}^{14}{ }^{14}$ In view of the lower density of the stable $\gamma$ form as compared to the metastable $\alpha$-form ( 1.37 and $1.42 \mathrm{~g} /$ $\mathrm{cm}^{3}$, respectively), the reactivity difference has been attributed to different molecular packing in the polymorphs, as the $\alpha$ form exposes carboxylic acid groups at the faces that can be easily accessed by ammonia. As amorphous materials have no long-range order and a large number of local packing motifs, 
these factors cannot to be invoked to explain the reactivity of different glasses. Instead, glass density appears to play a key role in determining reactivity with atmospheric gases. Physical aging and slow cooling from the liquid also increase glass density, but the density increase is much smaller than that observed by PVD; only small changes in reactivity would thus be anticipated for glasses prepared by these routes.

We note that many properties of PVD glasses are controlled by the substrate temperature during deposition. In addition to density, the enthalpy (packing energy) and birefringence (anisotropic molecular orientation) also vary considerably. ${ }^{17,18,21}$ Although we emphasize the correlation between chemical stability and density, the correlation between chemical stability and enthalpy is also high, and provides an alternate perspective: low energy glasses have high chemical stability. On the other hand, the chemical stability poorly correlates with the birefringence, with the birefringence changing sign at $T_{\text {sub }}=253 \mathrm{~K}$.

In the following three sections, we examine the influence of key experimental variables on the reaction rate between ammonia and indomethacin glasses, and then in Section 3.5 we present a quantitative model of the reaction kinetics consistent with this data.

3.2. Influence of Ammonia Concentration on Reactivity. To test the generality of our observation of reduced reaction rates in PVD glasses, we performed experiments using a higher concentration of ammonia gas. Figure 3 shows results

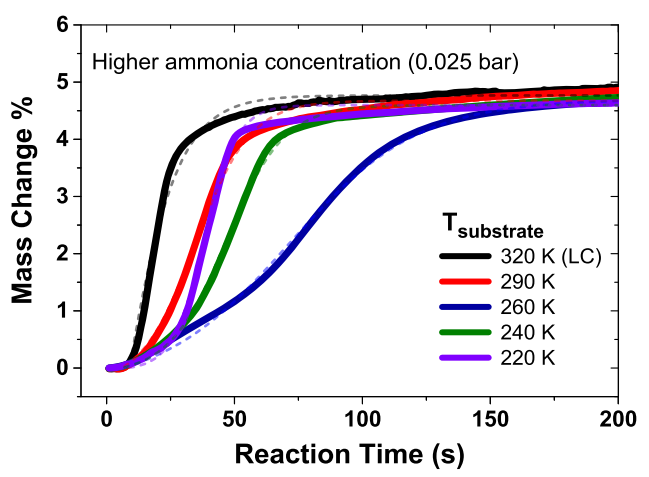

Figure 3. Mass increase for $100 \mathrm{~nm}$ glassy films of indomethacin reacting with 0.025 bar ammonia (in nitrogen) as a function of time at $295 \mathrm{~K}$. The black curve represents the LC glass and the colored curves represent glasses vapor-deposited at the indicated substrate temperatures. Dashed curves represent fits to a reaction model for each sample (see Section 3.5).

for PVD and LC glasses reacting with 0.025 bar ammonia at $295 \mathrm{~K}$. After exposure to ammonia gas, mass increases for all glasses, with the LC glass reacting faster than PVD samples. After $200 \mathrm{~s}$ had elapsed, the reaction was close to completion in all the glasses. Reactivity was quantified by the reaction halflife, as shown by Figure S3 in the Supporting Information. For this higher ammonia concentration, the reaction half-life is increased by about a factor of 3 for the most stable indomethacin glass in comparison with the LC glass. Instantaneous reaction rates for the PVD and LC glasses also support the conclusion of high chemical stability for PVD glasses at both ammonia pressures (Figure S4). Also shown in Figure 3 are fits to the experimental data, using the model to be described in Section 3.5.
3.3. Influence of Gas Diffusion on Reaction Half-Life. Given that indomethacin crystals react with ammonia gas via a reaction front that moves through the crystal at a constant velocity, ${ }^{14}$ it is important to understand the influence of sample thickness on the ammonia reaction with indomethacin glasses. Reaction via a front mechanism would result in a reaction time that increases linearly with film thickness. For indomethacin glasses, we found only a small influence of film thickness on the reaction half-life. These tests were performed on 100 and 300 $\mathrm{nm}$ films reacting with 0.025 bar ammonia at $295 \mathrm{~K}$. Half-lives for the reactions are summarized in Figure 4. For the most

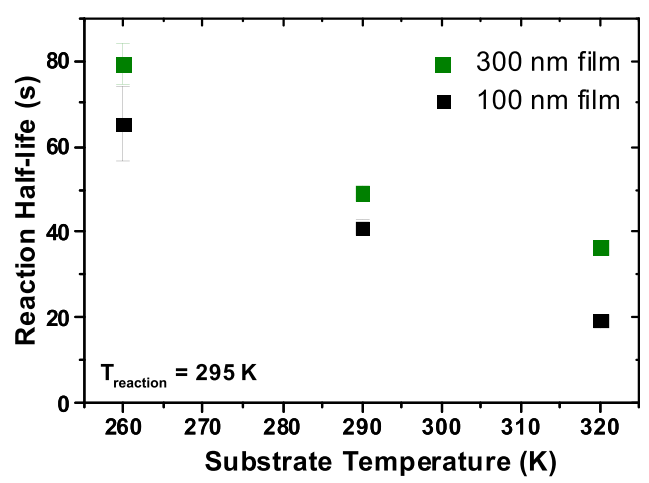

Figure 4. Reaction half-lives of indomethacin glasses for films of different thicknesses, for reaction with 0.025 bar ammonia. These results indicate that the reaction does not occur by a propagating front and is not diffusion-limited.

stable glass, the reaction half-life only increases by about $20 \%$ as the film thickness increases by a factor of 3 . These results indicate that the reaction of indomethacin glasses with ammonia gas, in contrast to the results obtained for crystalline indomethacin, is not controlled by a constant-velocity reaction front that initiates at the free surface.

For reactions between thick solids and gases, diffusion of the gas into the solid sometimes limits the rate of the reaction. The experimental results in Figure 4 allow us to conclude that diffusion does not limit the reaction rate in the glassy indomethacin films studied here. If we assume that the diffusion of ammonia into glassy thin films follows the Fickian model, then the time required to equilibrate the gas content within a film should scale with the film thickness squared. However, in Figure 4, a factor of 3 increase in thickness only yields a reaction time difference by approximately $20 \%$ for the most stable glass, far less than the factor of 9 increase expected from a diffusion-controlled mechanism. Consistent with this conclusion, Dawson and co-workers have measured the diffusion coefficient of water in PVD glasses of indomethacin to be roughly $10^{-9} \mathrm{~cm}^{2} / \mathrm{s}^{27}$ Assuming that ammonia and water have similar diffusion coefficients, the diffusion of ammonia into a $300 \mathrm{~nm}$ film should be fast (less than $1 \mathrm{~s}$ ) compared to the half-life of the indomethacin-ammonia reactions. The use of thin films in these experiments allows us to avoid the influence of the diffusion factor and thus directly characterize the influence of the glass structure on the kinetics of the solidgas reaction.

3.4. Influence of Reaction Temperature on the Reaction Rate. The influence of reaction temperature on the reactivity of indomethacin glasses with ammonia was also investigated and this provides a key insight about how the glass structure influences the rate of the reaction. As shown in 
Figure 5, for both the LC $\left(T_{\text {sub }}=320 \mathrm{~K}\right)$ and PVD glass, the reaction becomes faster at the lower temperature. (Reaction

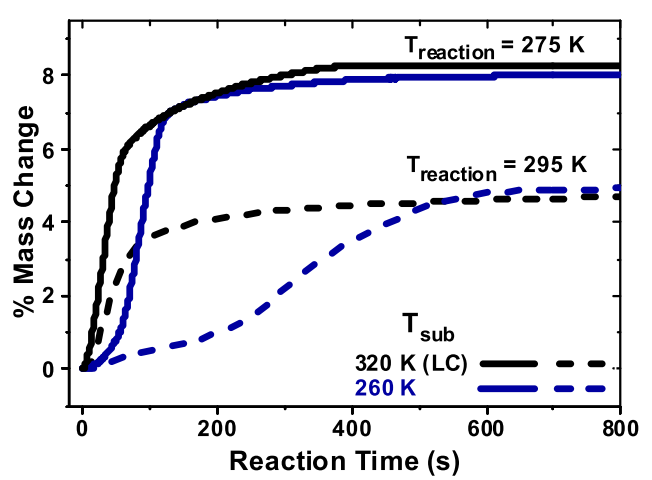

Figure 5. Mass increase for $100 \mathrm{~nm}$ indomethacin glasses reacting with 0.0076 bar ammonia as a function of time at different temperatures. Solid lines are results for the reaction at $275 \mathrm{~K}$; dashed lines are results obtained at $295 \mathrm{~K}$.

half-lives are shown in Figure S6.) The faster reaction at the lower temperature is naturally explained by the increased solubility of ammonia in amorphous indomethacin. At lower temperature, ammonia solubility is generally expected to increase in molecular solids. ${ }^{28,29}$ Consistent with this view, the maximum mass gain for the measurement at $275 \mathrm{~K}$ is about $8 \%$ at the steady-state shown in Figure 5, indicating substantial dissolution of molecular ammonia in the ammonium indomethacin salt.

3.5. Quantitative Model Analysis of Chemically Stable Glasses. In this section, we develop a simple model that provides a quantitative description of the kinetics of the reaction between indomethacin glasses and ammonia gas. As shown in Figure 2, the high density of the glass is clearly important in slowing the rate of the reaction. High glass density might impede the reaction in two different ways. First, the dense packing of surrounding molecules might destabilize the transition state and the reaction products, relative to the unreacted indomethacin; in this view, a well-packed glass disfavors the molecular rearrangements required to readily form the products. In support of this idea, we showed recently that the photochemical decarboxylation of indomethacin was roughly twice as slow in the densest PVD glass in comparison to the LC glass. ${ }^{30}$ Figure 5 suggests a second effect that may impede the reaction, that dense packing lowers the solubility of ammonia in amorphous indomethacin; the reaction will naturally proceed more slowly if the concentration of ammonia in the glass is lower. (Recall that our experiments control the partial pressure of ammonia above the glass, not the concentration of ammonia in the glass.) A previous study compared the uptake of water vapor by LC and PVD glasses of indomethacin, finding that solubility of water vapor decreased by a factor of 5 in the densest PVD glass. ${ }^{27}$ The decrease in gas solubility is qualitatively explained by a decrease in the space available to the gas (free volume) in the high-density PVD glass. If the solubility of ammonia decreases comparably to that of water vapor in PVD indomethacin, then we should expect a significant suppression of chemical reactivity with ammonia, in accordance with what we observe in experiments. Unfortunately, the solubility of ammonia in indomethacin glasses cannot be easily measured independently of the reaction between the species.
The two explanations above, direct inhibition of the reaction and diminished solubility of ammonia, have in common that their impact on the overall reaction kinetics should diminish as the reaction proceeds. This suggests a model of the reaction kinetics in which the rate constant depends upon the glass structure, changing smoothly from the initial rate $\left(k_{\text {initial }}\right)$ controlled by the structure of the as-deposited glass to the final rate $\left(k_{\text {final }}\right)$ controlled by the structure of the ammonium salt. The instantaneous rate constant $k$ obeys

$$
k(\phi)=\phi \cdot k_{\text {initial }}+(1-\phi) \cdot k_{\text {final }}
$$

where $\phi$ is the fraction of unreacted indomethacin. No matter which effect is dominant, the initial reaction rate should be slow and the reaction rate should increase as the initially wellpacked glass of indomethacin is replaced by the amorphous salt, until the depletion of indomethacin forces the rate to slow again. This is precisely the behavior shown in Figures 1, 3, and 5 (and most directly in Figure S4).

We further assume that the reaction between indomethacin and ammonia is first order in each component. With this assumption, the time-dependent increase in mass $(m)$ because of the reaction can be written in the following way

$$
m=A \cdot \frac{R \cdot \mathrm{e}^{k_{\text {final }} \cdot t}-R}{R \cdot \mathrm{e}^{-k_{\text {final }} \cdot t}-R+1}
$$

here $A$ is the maximum mass increase at steady-state, $k_{\text {initial }}$ is the initial rate constant, $k_{\text {final }}$ is the final rate constant, and $R=$ $k_{\text {initial }} / k_{\text {final }}$. The value of the initial rate constant $k_{\text {initial }}$ indicates the chemical stability of glasses reacting with ammonia at a very early stage of the reaction. Additional details are provided in the Supporting Information. We note that our model is conceptually similar to a model for solid state reactions developed by Shalaev, et al. ${ }^{31}$

Adjusting the three parameters in eq 3 provides a reasonably good fit to the experimental data, as indicated by the dashed curves in Figure 3, and a clear molecular interpretation. The fits are seen to follow a sigmoidal profile that closely reproduces the experimental data, where the reaction rates are low at the beginning and the end but rapid in between. Physically, the initial reaction rate is low because the high density of the PVD glass impedes the reaction. As the reaction proceeds, the high-density glass is disrupted by the reaction and the remaining indomethacin can react more quickly. Finally, the reaction rate decreases again because of depletion of the indomethacin.

The chemical stability of vapor-deposited indomethacin glasses relative to the highly disrupted glass present at the end of the reaction is characterized by the ratio of the initial and final rate constants $\left(k_{\text {initial }} / k_{\text {final }}\right)$, as summarized in Figure 6. In their first exposure to ammonia gas, glasses vapor-deposited near $240 \mathrm{~K}$ show the smallest values of this ratio, consistent with the initial reaction being slowest for high density glasses. As shown in Figure 6, experiments performed at the higher ammonia concentration ( 0.025 bar) are reasonably consistent with experiments at $0.0076 \mathrm{bar}$; for both concentrations, the glass deposited at $240 \mathrm{~K}$ has $k_{\text {initial }} / k_{\text {final }} \approx 0.01$. We interpret this to indicate that the instantaneous rate constant for the reaction of this PVD glass is about 100 times smaller than for the LC glass, with the assumption that $k_{\text {final }}$ of the PVD glass is equal to the LC glass. Consistent with this interpretation, we note that $k_{\text {initial }} / k_{\text {final }} \approx 1$ for a LC glass (deposited at $320 \mathrm{~K}$ ). Also shown in Figure 6 are the values of $k_{\text {initial }} / k_{\text {final }}$ for the 


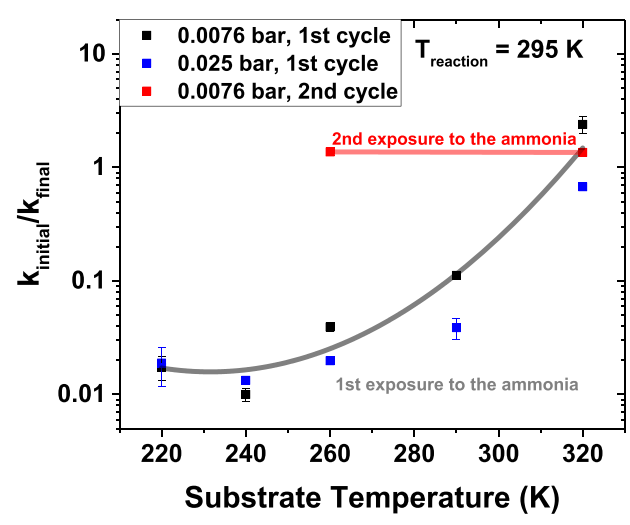

Figure 6. Ratio of the initial rate constant to the final rate constant for indomethacin glasses vapor-deposited at various substrate temperatures and measured at different reaction conditions. For the most stable glasses, the rate constant is 100 times smaller at the start of the reaction than at the end, consistent with the view that a dense initial glass structure strongly impedes the reaction. Red and grey curves are guides to the eye.

second exposure to ammonia gas (see Figure S5). The observation that this ratio is independent of substrate temperature is consistent with the conclusion that glassy packing of indomethacin is completely disrupted in the initial exposure to ammonia, as demonstrated in the inset of Figure 1.

The success of the phenomenological model employed in this section is consistent with either of the two proposed mechanisms for inhibition: direct inhibition of the reaction by tight packing and diminished solubility of ammonia. We are confident that reduced ammonia solubility plays a large role in explaining the reaction inhibition in dense glasses. This conclusion is supported both by the very large impact of solubility indicated by Figure 5, and by the previous observation that water solubility is five times smaller in dense PVD glasses of indomethacin in comparison to the LC glass. $^{27}$ Given the observation that the photochemical decarboxylation of indomethacin glasses is slowed by a factor of two in dense indomethacin glasses, it also seems likely that direct inhibition of the reaction by tight packing is playing a role.

\section{CONCLUSIONS}

We have shown for the first time that the rate of reaction between an organic glass and an atmospheric gas can be significantly modulated by controlling the glass packing. By optimizing the substrate temperature during PVD, we have created glasses of indomethacin that react an order of magnitude more slowly than the LC glass, which is an unprecedented large effect in glassy solids. Previous work on crystals indicated the important influence of the local packing environment, with different crystal polymorphs reacting at different rates. Analogously, PVD provides a range of different glass structures to investigate the principles of gas-glass reactions. In contrast with crystals, chemical stability in glassy solids is observed to correlate reasonably with density, where tighter packing results in a slower reaction. Comparing the reaction in films of different thicknesses, we eliminate the possibility that the reaction is limited by gas diffusion or associated with a constant velocity reaction front. We attribute the enhanced chemical stability in high density glasses to the diminished solubility of ammonia and direct inhibition of the reaction by the dense packing of surrounding molecules. The use of PVD for glass preparation in this work provided access to high glass densities that cannot be achieved by other methods, thus allowing the correlation between glass density and the reaction rate to be easily observed.

The enhanced chemical stability reported here for vapordeposited indomethacin is expected to be quite general for organic molecules prepared by PVD and this has potential technological significance. So far, more than 30 organic molecules have been made into high-density glasses by $\mathrm{PVD}^{17}$ and many of these molecules are used in OLEDs. OLED devices require encapsulation for the protection of amorphous light emission layers from water and oxygen. Given that OLEDs are made by PVD, our work suggests that optimizing the substrate temperature during deposition to produce the densest glass would lead to a greater intrinsic resistance to device degradation initiated by environmental gases. Consistent with this view, vapor-deposited OLED layers which were optimized to maximize density were recently shown to increase the device lifetime. ${ }^{11,32}$

\section{ASSOCIATED CONTENT}

\section{Supporting Information}

The Supporting Information is available free of charge on the ACS Publications website at DOI: 10.1021/acs.jpcb.9b08360.

Additional data and discussions (PDF)

\section{AUTHOR INFORMATION}

\section{Corresponding Author}

*E-mail: yqiu@chem.wisc.edu.

ORCID $\odot$

Yue Qiu: 0000-0001-8813-2646

M. D. Ediger: 0000-0003-4715-8473

\section{Author Contributions}

All the authors have given approval to the final version of the manuscript.

Notes

The authors declare no competing financial interest.

\section{ACKNOWLEDGMENTS}

This work was supported by the U.S. Department of Energy (DOE), Office of Basic Energy Sciences (BES), Division of Materials Sciences and Engineering, Award DE-SC0002161. We thank Lian Yu and George Zografi for helpful discussions, and Kushal Bagchi for help with X-ray scattering measurements.

\section{ABBREVIATIONS}

PVD, physical vapor deposition; $T_{\mathrm{g}}$, glass transition temperature; QCM, quartz crystal microbalance

\section{REFERENCES}

(1) Hancock, B. C.; Zografi, G. Characteristics and Significance of the Amorphous State in Pharmaceutical Systems. J. Pharm. Sci. 1997, $86,1-12$.

(2) Laitinen, R.; Löbmann, K.; Strachan, C. J.; Grohganz, H.; Rades, T. Emerging Trends in the Stabilization of Amorphous Drugs. Int. J. Pharm. 2013, 453, 65-79.

(3) Yokoyama, D. Molecular Orientation in Small-Molecule Organic Light-Emitting Diodes. J. Mater. Chem. 2011, 21, 19187-19202.

(4) Kim, S.-Y.; Jeong, W.-I.; Mayr, C.; Park, Y.-S.; Kim, K.-H.; Lee, J.-H.; Moon, C.-K.; Brütting, W.; Kim, J.-J. Organic Light-Emitting 
Diodes with 30\% External Quantum Efficiency Based on a Horizontally Oriented Emitter. Adv. Funct. Mater. 2013, 23, 38963900.

(5) Suzuki, F.; Kubo, S.; Fukushima, T.; Kaji, H. Effects of Structural and Energetic Disorders on Charge Transports in Crystal and Amorphous Organic Layers. Sci. Rep. 2018, 8, 5203.

(6) Dengale, S. J.; Grohganz, H.; Rades, T.; Löbmann, K. Recent Advances in Co-Amorphous Drug Formulations. Adv. Drug Delivery Rev. 2016, 100, 116-125.

(7) Duddu, S. P.; Weller, K. Importance of Glass Transition Temperature in Accelerated Stability Testing of Amorphous Solids: Case Study Using a Lyophilized Aspirin Formulation. J. Pharm. Sci. 1996, 85, 345-347.

(8) Ohtake, S.; Shalaev, E. Effect of Water on the Chemical Stability of Amorphous Pharmaceuticals: I. Small Molecules. J. Pharm. Sci. 2013, 102, 1139-1154.

(9) Liao, L. S.; Sun, X. H.; Cheng, L. F.; Wong, N. B.; Lee, C. S.; Lee, S. T. Ambient Effect on the Electronic Structures of Tris-(8Hydroxyquinoline) Aluminum Films Investigated by Photoelectron Spectroscopy. Chem. Phys. Lett. 2001, 333, 212-216.

(10) Knox, J. E.; Halls, M. D.; Hratchian, H. P.; Bernhard Schlegel, H. Chemical Failure Modes of Alq3-Based Oleds: Alq3 Hydrolysis. Phys. Chem. Chem. Phys. 2006, 8, 1371-1377.

(11) Esaki, Y.; Komino, T.; Matsushima, T.; Adachi, C. Enhanced Electrical Properties and Air Stability of Amorphous Organic Thin Films by Engineering Film Density. J. Phys. Chem. Lett. 2017, 8, 5891-5897.

(12) Miller, R. S.; Curtin, D. Y.; Paul, I. C. Reactions of Molecular Crystals with Gases. I. Reactions of Solid Aromatic Carboxylic Acids and Related Compounds with Ammonia and Amines. J. Am. Chem. Soc. 1974, 96, 6329-6334.

(13) Miller, R. S.; Paul, I. C.; Curtin, D. Y. Reactions of Molecular Crystals with Gases. Ii. X-Ray Structure of Crystalline 4Chlorobenzoic Acid and the Anisotropy of Its Reaction with Ammonia Gas. J. Am. Chem. Soc. 1974, 96, 6334-6339.

(14) Chen, X.; Morris, K. R.; Griesser, U. J.; Byrn, S. R.; Stowell, J. G. Reactivity Differences of Indomethacin Solid Forms with Ammonia Gas. J. Am. Chem. Soc. 2002, 124, 15012-15019.

(15) Byrn, S. R.; Pfeiffer, R. R.; Stowell, J. G. Solid-State Chemistry of Drugs, 2nd ed.; SSCI, Inc.: 3065 Kent Avenue, West Lafayette, Indiana 47906-1076, 1999.

(16) Swallen, S. F.; Kearns, K. L.; Mapes, M. K.; Kim, Y. S.; McMahon, R. J.; Ediger, M. D.; Wu, T.; Yu, L.; Satija, S. Organic Glasses with Exceptional Thermodynamic and Kinetic Stability. Science 2007, 315, 353-356.

(17) Ediger, M. D. Perspective: Highly Stable Vapor-Deposited Glasses. J. Chem. Phys. 2017, 147, 210901.

(18) Dalal, S. S.; Fakhraai, Z.; Ediger, M. D. High-Throughput Ellipsometric Characterization of Vapor-Deposited Indomethacin Glasses. J. Phys. Chem. B 2013, 117, 15415-15425.

(19) Kearns, K. L.; Swallen, S. F.; Ediger, M. D.; Wu, T.; Sun, Y.; Yu, L. Hiking Down the Energy Landscape: Progress toward the Kauzmann Temperature Via Vapor Deposition. J. Phys. Chem. B 2008, 112, 4934-4942.

(20) Walters, D. M.; Richert, R.; Ediger, M. D. Thermal Stability of Vapor-Deposited Stable Glasses of an Organic Semiconductor. J. Chem. Phys. 2015, 142, 134504.

(21) Ràfols-Ribé, J.; Gonzalez-Silveira, M.; Rodríguez-Tinoco, C.; Rodríguez-Viejo, J. The Role of Thermodynamic Stability in the Characteristics of the Devitrification Front of Vapour-Deposited Glasses of Toluene. Phys. Chem. Chem. Phys. 2017, 19, 11089-11097.

(22) Qiu, Y.; Antony, L. W.; de Pablo, J. J.; Ediger, M. D. Photostability Can Be Significantly Modulated by Molecular Packing in Glasses. J. Am. Chem. Soc. 2016, 138, 11282-11289.

(23) Banda, L.; Alcoutlabi, M.; McKenna, G. B. Errors Induced in Quartz Crystal Mass Uptake Measurements by Nongravimetric Effects: Considerations Beyond the Eernisse Caution. J. Polym. Sci., Part B: Polym. Phys. 2006, 44, 801-814.
(24) Muraoka, S.; Kiyohara, Y.; Oue, H.; Higashimoto, S. A Co2 Sensor Using a Quartz Crystal Microbalance Coated with a Sensitive Membrane. Electron. Commun. 2014, 97, 60-66.

(25) Sauerbrey, G. Verwendung Von Schwingquarzen Zur Wägung Dünner Schichten Und Zur Mikrowägung. Z. Phys. 1959, 155, 206222.

(26) Dawson, K. J.; Zhu, L.; Yu, L.; Ediger, M. D. Anisotropic Structure and Transformation Kinetics of Vapor-Deposited Indomethacin Glasses. J. Phys. Chem. B 2011, 115, 455-463.

(27) Dawson, K. J.; Kearns, K. L.; Ediger, M. D.; Sacchetti, M. J.; Zografi, G. D. Highly Stable Indomethacin Glasses Resist Uptake of Water Vapor. J. Phys. Chem. B 2009, 113, 2422-2427.

(28) Hancock, B. C.; Dalton, C. R. The Effect of Temperature on Water Vapor Sorption by Some Amorphous Pharmaceutical Sugars. Pharm. Dev. Technol. 1999, 4, 125-131.

(29) Stevens, K. A.; Smith, Z. P.; Gleason, K. L.; Galizia, M.; Paul, D. R.; Freeman, B. D. Influence of Temperature on Gas Solubility in Thermally Rearranged (Tr) Polymers. J. Membr. Sci. 2017, 533, 7583.

(30) Qiu, Y.; Dalal, S. S.; Ediger, M. D. Vapor-Deposited Organic Glasses Exhibit Enhanced Stability against Photodegradation. Soft Matter 2018, 14, 2827-2834.

(31) Shalaev, E. Y.; Byrn, S. R.; Zografi, G. Single-Phase and Heterophase Solid-State Chemical Kinetics of Thermally Induced Methyl Transfer in Tetraglycine Methyl Ester. Int. J. Chem. Kinet. 1997, 29, 339-348.

(32) Ràfols-Ribé, J.; Will, P.-A.; Hänisch, C.; Gonzalez-Silveira, M.; Lenk, S.; Rodríguez-Viejo, J.; Reineke, S. High-Performance Organic Light-Emitting Diodes Comprising Ultrastable Glass Layers. Sci. Adv. 2018, 4, No. eaar8332. 